\title{
Skin colour and bilirubin in neonates
}

\author{
A KNUDSEN AND R BRODERSEN
}

Department of Obstetrics and Gynaecology, County Hospital, Hjørring and Institute of Medical Biochemistry, University of Aarhus, Denmark

SUMmARY The correlation between the yellow colour of the skin and serum bilirubin concentration, reserve albumin concentration, and $\mathrm{pH}$ was investigated in 76 icteric neonates. Significant linear correlation existed between yellow colour of the skin and serum bilirubin concentration, reciprocal of the reserve albumin concentration, and the squared hydrogen ion concentration. Furthermore, the basic yellowness of the skin at birth correlated linearily with the yellow colour of the skin measured when the child became jaundiced.

The results support the proposed hypothesis that bilirubin is transferred from plasma to skin through two different mechanisms: (a) leakage of bilirubin-albumin complexes into extravascular spaces and (b) precipitation of bilirubin acid in phospholipid membranes. The latter mechanism suggests that measurement of the yellow colour of the skin may be a better predictor of brain damage than the serum bilirubin concentration and thus be of clinical utility. Measurement of the yellow colour of the skin as a method of obtaining serum bilirubin concentration is unreliable.

Measurement of the yellow colour of the skin, so called transcutaneous bilirubinometry, has been introduced as an alternative to determination of bilirubin in serum of neonates. ${ }^{12}$ Several authors have found a good correlation between the instrument reading and the serum bilirubin concentration. ${ }^{1-4}$ Even though a non-invasive method offers several advantages over an invasive procedure widespread use of the bilirubinometer has not been seen. ${ }^{5}$ It may be noted in this connection that the reliability of serum bilirubin concentration as a risk indicator of bilirubin encephalopathy is questioned from different quarters. ${ }^{7}$ Also, other factors than serum bilirubin concentration influence the readings.

The present investigation was undertaken in order to elucidate the meaning of measurements of the yellow colour of the skin, after consideration of possible bilirubin transfer mechanisms between blood plasma and skin tissue. The clinical utility of the method will be discussed in the light of the results obtained.

We hypothesise that bilirubin may be transferred to the skin through two different mechanisms: (a) by leakage of bilirubin-albumin from plasma into extravascular spaces and (b) by precipitation of bilirubin acid in phospholipid membranes.

The first of these two possibilities is an obvious one. A considerable amount of serum albumin is found outside the blood stream, the extravascular albumin pool constituting about $60 \%$ of the total in adults. ${ }^{8}$ As bilirubin is bound with high affinity to albumin and as the intravascular and extravascular pools of the protein exchange, albeit slowly, ${ }^{9}$ it can be presumed that the extravascular albumin becomes loaded with bilirubin. The skin contains considerable amounts of albumin. It is conceivable, therefore, that in the case of hyperbilirubinemia the skin will assume a yellow colour due to its content of bilirubin-albumin.

The second mechanism hypothesised above, precipitation of bilirubin acid, is likely to be active in icteric infants. It has been found that blood plasma in such cases is supersaturated with bilirubin acid, ${ }^{10}$ and experiments in vitro have shown that the pigment promptly precipitates in phospholipid membranes when these are in contact with a supersaturated bilirubin solution. ${ }^{11}$

Other mechanisms appear unlikely at our present state of knowledge. The possibility that bilirubin should bind to collagen or elastin has been investigated and gave negative results ( $R$ Brodersen, unpublished observations). Likewise, dissolution of bilirubin in fat of adipose tissue has not been shown and seems remote after experiments in vitro as well as in vivo. ${ }^{12} 13$

If bilirubin in the skin tissue is present as bilirubin-albumin, then the yellow colour of the skin 
will be correlated to the plasma bilirubin concentration. If deposition of bilirubin acid is involved we further expect a negative correlation to the concentration of reserve albumin for binding of bilirubin and a negative correlation to plasma $\mathrm{pH}$. It is the aim of the present paper to investigate whether such correlations exist and to discuss the possible bearing of these on bilirubin transfer mechanisms to the skin as well as to other tissues.

\section{Theory}

If the plasma concentration of bilirubin-albumin is $B$, and the first order rate constant for transfer of this complex to extravascular spaces in the skin is $\mathrm{k}_{\mathrm{p}+}$ then the rate of transfer of bilirubin-albumin is $\mathrm{Bk}_{\mathrm{p}+}$. The rate of the reverse process, transfer of bilirubin-albumin from skin to plasma is then $B^{\prime} k_{p}$, the bilirubin-albumin concentration in the extravascular spaces multiplied by the rate constant for the reverse transport. Deposition of bilirubin acid occurs through the process:

$$
\mathrm{AB}^{2-}+2 \mathrm{H}^{+} \rightleftarrows \mathrm{BH}_{2}+\mathrm{A}
$$

The bilirubin-albumin complex, in which bilirubin is present as the dianion, combines with two hydrogen ions in the plasma and deposits a molecule of solid bilirubin acid in the capillary wall; one molecule of albumin without bilirubin is released and remains in the blood stream. The rate of bilirubin transfer through this process may be proportional to the concentration of bilirubinalbumin, $\mathrm{B}$, and to the squared hydrogen concentration and can thus be expressed as $\mathrm{B} 10^{-2 \mathrm{pH}_{\mathrm{H}}} \mathrm{k}_{\mathrm{A}+}$, when the rate constant is $\mathrm{k}_{\mathrm{A}}$ and as the hydrogen ion concentration is $10^{-\mathrm{pH}}$.

The reverse process, dissolution of bilirubin acid by reaction with albumin, takes place with release of two hydrogen ions and formation of one molecule of the bilirubin-albumin complex in the plasma. The rate of this process is presumably $\mathrm{aB}^{\prime \prime} \mathrm{k}_{\mathrm{A}_{-}}$, proportional to the reserve albumin concentration for binding of bilirubin, a. The amount of bilirubin acid per volume of skin tissue is $B^{\prime \prime}$. (We have disregarded the fact that albumin can bind more than one bilirubin dianion per molecule of the protein. Consideration of multiple bilirubin binding results in a more complicated deduction but the same final expression for the transfer rate, when a is used for the reserve albumin concentration, as previously defined. ${ }^{14}$ )

The net rate of bilirubin deposition in the skin is further influenced by elimination of bilirubin after photoisomerisation and possibly also by oxidation with bilirubin oxidase. ${ }^{15}$ Let us denote the rates of these two processes by $v_{L}$ and $v_{0 x}$ respectively. The rate of bilirubin deposition in the skin is then:

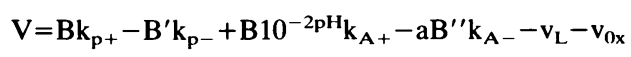

The intensity of the yellow colour of the skin at a given point of time, $t_{1}$, is considered to consist of a constant basic colour intensity, $\mathrm{Y}_{0}$, which was present at the time of birth, $\mathrm{t}_{0}$, and a contribution accumulated over the time. The total colour intensity is then:

$$
\mathrm{Y}=\mathrm{Y}_{0}+\iint_{\mathrm{t}_{0}}^{\mathrm{t}_{1}} \mathrm{Vdt}
$$

Equation (3) cannot be solved numerically in general terms, but an understanding of the dynamics involved may be obtained as follows. If jaundice is in an early phase of building up, we may consider B and $\mathrm{pH}$ approximately constant with time and $\mathrm{B}^{\prime}$, $B^{\prime \prime}, v_{L}$ and $v_{0 x}$ as zero, and obtain:

$$
\mathrm{Y}=\mathrm{Y}_{0}+\mathrm{B}\left(\mathrm{k}_{\mathrm{P}+}+10^{-2 \mathrm{pH}} \mathrm{k}_{\mathrm{A}+}\right) \quad\left(\mathrm{t}_{1}-\mathrm{t}_{0}\right)
$$

In an early phase of jaundice we may thus expect to see the colour intensity of the skin increase linearly with time. If our hypothesised mechanisms of bilirubin transfer are correct we should observe that the colour intensity of the skin is positively correlated to the plasma bilirubin concentration, $\mathrm{B}$, and negatively correlated to plasma $\mathrm{pH}$.

Later on, as bilirubin accumulates in the tissue, the rates of reverse transport of bilirubin-albumin and of bilirubin acid dissolution increase. Also the rates of photoelimination and of bilirubin oxidation may become significant. A maximum level of jaundice may be reached, in healthy infants usually around the fourth day of life. The maximal colour intensity will depend upon the initial rate of colour increase and further be inversely related to the rate of bilirubin acid dissolution. If our hypothesis is correct, the maximal level of jaundice should therefore be positively correlated to the plasma bilirubin concentration and negatively to the plasma $\mathrm{pH}$. A high reserve albumin concentration, a, will at this stage cause an increase of bilirubin acid dissolution and thus tend to decrease the maximal level.

An equilibrium may be established if the velocities of photoelimination and of enzymatic oxidation remain insignificant. In this case both the transport of bilirubin-albumin and the deposition of bilirubin acid will be in equilibrium, with equal rates of transport in and out of the tissue, We then have:

$$
\mathrm{Bk}_{\mathrm{p}+}=\mathrm{B}^{\prime} \mathrm{k}_{\mathrm{p}-} \text { and } \mathrm{B} 10^{-2 \mathrm{pH}} \mathrm{k}_{\mathrm{A}+}=\mathrm{aB}^{\prime \prime} \mathrm{k}_{\mathrm{A}-}
$$

The skin colour consists of the basic colour, the 
bilirubin-albumin, and the bilirubin acid present in the tissue:

$$
\mathrm{Y}=\mathrm{Y}_{0}+\mathrm{B}^{\prime}+\mathrm{B}^{\prime \prime}
$$

$B^{\prime}$ and $B^{\prime \prime}$ are isolated from equation (5) and entered into equation (6).

$$
\mathrm{Y}=\mathrm{Y}_{0}+\mathrm{B}\left(\frac{\mathrm{k}_{\mathrm{p}+}}{\mathrm{k}_{\mathrm{p}-}}+\frac{10^{-2 \mathrm{pH}_{\mathrm{k}}} \mathrm{k}_{\mathrm{A}+}}{\mathrm{ak}_{\mathrm{A}-}}\right)
$$

If our hypothesis is correct we expect, in a state of equilibrium between plasma and skin, to observe that the colour intensity of the skin is linearily related to the plasma bilirubin concentration, $B$, and further linearily related to the squared hydrogen ion concentration, $10^{-2 \mathrm{pH}}$, and finally to the reciprocal of the reserve albumin concentration, 1/a.

In summary, the intensity of the yellow colour of the skin should be positively correlated to three indices: the plasma bilirubin concentration, the squared hydrogen ion concentration, and the reciprocal of the reserve albumin concentration. This should form a suitable basis for testing the hypothesis. It is obvious, however, that strict correlations to these indices cannot be expected. As seen from equations (4) and (7), the yellow colour varies with several factors, to different degrees under different circumstances. Weak correlations, if significant, must be accepted as positive findings.

\section{Subjects and methods}

The study population consisted of 76 healthy newborn babies admitted to the newborn nursery of Hjørring hospital. All infants were of more then 36 weeks' gestation. None of the infants suffered from haemolytic anaemia or had mothers who suffered from diabetes. No infants had been exposed to phototherapy or exchange transfusion before measurement. All measurements were obtained during the first five days after birth.

The readings of the yellow colour of the skin were taken with the Air Shields Jaundice Meter (Minolta Camera Co). The Air Shields Jaundice Meter is a hand held instrument that employs fibre optic techniques to illuminate the skin and subcutaneous tissue and then spectrophotometrically analyses the intensity of the yellow colour. The meter was used and tested daily as recommended by the manufacturer. ${ }^{16}$

All infants within the study period who had visible jaundice were included after informed consent. A single set of data was obtained from each infant at the time when the child was initially observed to be icteric. At the time of blood sampling for serum bilirubin concentration determination two meter readings were obtained from the forehead, which was the site chosen because of convenience and accessibility. Furthermore, the cephalocaudal progression of neonatal jaundice with higher bilirubin values could lead to some reservations being held on the reliability of the more distal sites for meter readings. ${ }^{17}$ The average value of the two readings was obtained. The meter readings were performed by members of the nursing staff.

During a part of the study jaundice meter readings were obtained in all infants immediately after birth.

Blood samples were obtained by heel prick. The total bilirubin concentration was determined in capillary blood serum by a standard diazo method. ${ }^{18}$ As all neonates in the study were mature, between 2 and 5 days old, icteric, but otherwise clinically well without diseases in the biliary system or infection, the amount of conjugated bilirubin in the blood was probably around $1 \%$ of the total serum bilirubin concentration. ${ }^{19}$ Therefore, in the theoretical section, the concentration of conjugated bilirubin in serum was regarded as zero and the concentration of unconjugated bilirubin in serum was considered equal to the total serum bilirubin concentration. The reserve albumin concentration was measured using the ${ }^{14} \mathrm{C}$-MADDS (monoacetyldiamino-diphenylsulphone) method. ${ }^{14} \mathrm{pH}$ was determined by an ABL3 $\mathrm{pH} /$ blood-gas analyser (Radiometer).

\section{Results and conclusions}

The meter readings ranged from 10 to 23 with mean (SD) value of $16(2 \cdot 6)$. The mean serum bilirubin concentration for all infants was 185 (47) $\mu \mathrm{mol} / \mathrm{l}$ (range 68-282 $\mu \mathrm{mol} / \mathrm{l}$ ). The mean reserve albumin concentration was 129 (32) $\mu \mathrm{mol} / \mathrm{l}$ (range 71-212 $\mu \mathrm{mol} / \mathrm{l})$. Plasma $\mathrm{pH}$ ranged from $7 \cdot 30$ to $7 \cdot 48$. The mean value was $7.40(0.04)$.

Results of linear correlation analysis by the method of least squares are presented in the table. The correlation between serum bilirubin concentration and the yellow colour of the skin is pictured in the figure.

The yellow colour of the skin of icteric infants is thus correlated to the serum bilirubin concentration and inversely to the reserve albumin and $\mathrm{pH}$, and further to the basic colour intensity measured at birth.

During the icterometer reading the skin is compressed so that blood is removed from the capillaries. The yellow colour is due to bilirubin located extravascularly in the skin, and to some extent to the basic colour of the tissue. The correlation of the colour intensity with serum bilirubin concentration 
Table Results of linear correlation analysis

\begin{tabular}{|c|c|c|}
\hline Correlation & $\begin{array}{l}\text { Coefficient of } \\
\text { correlation }\end{array}$ & p Value \\
\hline $\mathrm{Y} v \mathrm{~B}$ & $0 \cdot 83$ & $<0.0001$ \\
\hline $\mathrm{Y} v 1 / \mathrm{a}$ & 0.43 & $<0.001$ \\
\hline $\mathrm{Y} v 10^{-2 \mathrm{pH}}$ & $0 \cdot 33$ & $<0.02$ \\
\hline $\mathrm{Y} v \mathrm{Y}_{0}$ & $0 \cdot 35$ & $<0.05$ \\
\hline B $v$ a & 0.02 & NS \\
\hline $\mathrm{B} v \mathrm{pH}$ & -0.12 & NS \\
\hline
\end{tabular}

$\mathrm{Y}$ is the yellow colour of the skin as measured by the jaundice meter, B the total serum bilirubin concentration, a the reserve albumin concentration, and $Y_{0}$ the basic yellowness of the skin at birth.

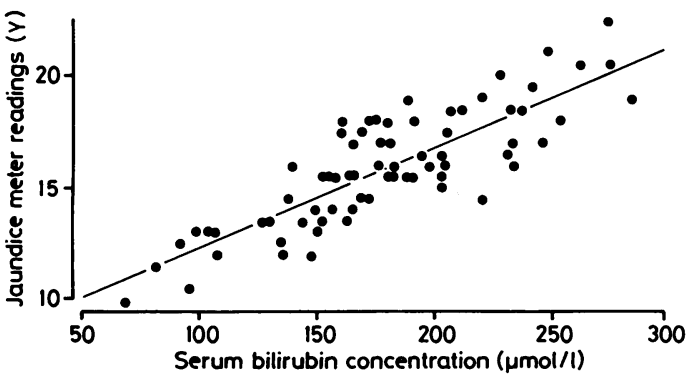

Figure Correlation between the jaundice meter readings and the serum bilirubin concentration in 76 newborn infants. Regression line: $Y=7 \cdot 75+0 \cdot 046 X$.

can be explained by the presence of bilirubinalbumin in extravascular spaces.

The inverse correlations to the reserve albumin concentration and $\mathrm{pH}$ can be understood as a result of bilirubin acid precipitation, as developed in the theoretical section. As the serum bilirubin concentration is not significantly related to reserve albumin or the plasma $\mathrm{pH}$, the effect of a low reserve albumin or a low $\mathrm{pH}$ cannot be explained as an indirect one, exerted through an effect of these indices on serum bilirubin concentration. It is likely, therefore, that a direct causal relationship exists between the yellow colour and a low reserve albumin and low $\mathrm{pH}$ and that bilirubin in the skin is in part present as precipitated bilirubin acid.

\section{Discussion}

Measuring the yellow colour of the skin is not a suitable method for obtaining the plasma bilirubin concentration as the yellow colour depends upon other factors - the reserve albumin concentration, plasma $\mathrm{pH}$, and the basic skin colour-in addition to the plasma bilirubin. According to the figure errors may amount to as much as $93 \mu \mathrm{mol} / \mathrm{l}$.
Furthermore, the plasma bilirubin concentration is not a suitable predictor of bilirubin encephalopathy. ${ }^{672021}$ Deposition of bilirubin in the newborn brain depends upon other factors than serum bilirubin concentration-for example, the presence of bilirubin displacing drugs,${ }^{22}$ acidosis ${ }^{23}{ }^{24}$ etc. It seems necessary, therefore, to discuss the utility of skin colour measurements in the management of icteric infants.

As shown above, it is likely that bilirubin is transferred to the icteric skin of neonates by two mechanisms; leakage of bilirubin-albumin into extravascular spaces and precipitation of bilirubin acid. The first of these mechanisms is probably not active in the brain because the central nervous system has no extravascular albumin space. ${ }^{25} \mathrm{We}$ cannot, therefore, expect that kernicterus should parallel jaundice of the skin. In the absence of acidosis the skin may become icteric without involvement of the brain, as seen in adults in cases of haemolytic icterus, etc. On the other hand if kernicterus develops in an infant due to high serum bilirubin, low reserve albumin, and acidosis, bilirubin acid will also be deposited in the skin. In consequence, it seems possible that the yellow colour of the skin is correlated with that of the brain, to the extent that bilirubin deposition in the skin has occurred by precipitation of bilirubin acid. If so, the intensity of the yellow colour of the skin may be a somewhat better predictor of brain damage than the serum bilirubin concentration and measurement of the colour of the skin may theoretically be of clinical utility.

We wish to thank Birgitte Bannebjerg Frederiksen for technical work, the nursing and laboratory staff for help in jaundice meter readings and serum bilirubin concentrations measurements, and Frede Nielsen for the drawing. This study was supported by Nordjyllands Amtskommunes Forskningsfond Grant No 2-16-30, and by Niels Jensens Forskningsfond Grant No 5-9-1986.

\section{References}

1 Yamanouchi I, Yamauchi Y, Igarashi I. Transcutaneous bilirubinometry: preliminary studies of noninvasive transcutaneous bilirubin meter in the Okayama National Hospital. Pediatrics 1980;65:195-202.

${ }^{2}$ Hegyi T, Hiatt IM, Indyk L. Transcutaneous bilirubinometry. Correlations in term infants. J Pediatr 1981;98:454-7.

3 Tan KL. Transcutaneous bilirubinometry in fullterm Chinese and Malay infants. Acta Paediatr Scand 1982;71:593-6.

${ }^{4}$ Hannemann RE. Evaluation of the Minolta bilirubin meter as a screening device in white and black infants. Pediatrics 1982;69:107-9.

5 Brown AK, Kim MH, Valencia G, Nuchpuckee P, Boyle G. Factors affecting the transcutaneous measurement of bilirubin: influence of race, gestational age, phototherapy and albumin binding capacity. In: Rubatelli FF, ed. Neonatal jaundice. New York: Plenum Publishing Corporation, 1984:95-109.

6 Lucey JF. Bilirubin and brain damage: a real mess. Pediatrics 1982;69:381-2.

${ }^{7}$ Kim MH, Yoon JJ, Sher J, Brown AK. Lack of predictive 
indices in kernicterus: a comparison of clinical and pathologic factors in infants with and without kernicterus. Pediatrics 1980;66:852-8.

${ }^{8}$ Katz J, Bonorris G, Sellers AL. Extravascular albumin in human tissues. Clinical Science and Molecular Medicine 1970;39:725-9.

9 Smith PC, Frank HA, Kasdon EJ, et al. Albumin uptake by skin, skeletal muscle and lung in living and dying patients. Ann Surg 1978;187:31-7.

10 Cashore WJ, Oh W, Brodersen R. Reserve albumin and bilirubin toxicity index in infant serum. Acta Paediatr Scand 1983;72:415-9.

"Eriksen EF, Danielsen H, Brodersen R. Bilirubin-liposome interaction: formation of a bilirubin dianion-phospholipid complex, followed by bilirubin acid aggregation. J Biol Chem 1981;256:4269-74.

12 Brodersen R. Bilirubin solubility and interaction with albumin and phospholipids. J Biol Chem 1979;254:2364-9.

13 Brodersen R. Localization of bilirubin pools in the nonjaundiced rat with a note on bilirubin dynamics in normal human adults and in Gilbert's syndrome. Scand J Clin Lab Invest 1972;30:95-106.

14 Brodersen R, Andersen S, Jacobsen C, et al. Determination of reserve albumin-equivalent for ligand binding, probing two distinct binding functions of the protein. Anal Biochem 1982;121:395-408.

15 Brodersen R, Bartels P. Enzymatic oxidation of bilirubin. Eur J Biochem 1969;10:468-73.

16 Anonymous. MinoltalAir-Shields Jaundice Meter 101 operators manual. Hatboro, Pennsylvania: Narco Scientific, Air-Shields Division, 1981

17 Ebbesen F. The relationship between the cephalo-pedal pro- gress of clinical icterus and the serum bilirubin concentration in newborn infants without blood type sensitization. Acta Obstet Gynecol Scand 1975;54:329-32.

${ }^{18}$ Doumas BT, Perry BW, Sasse EA, Straumfjord JV. Standardization in bilirubin assays: evaluation of selected methods and stability of bilirubin solutions. Clin Chem 1973;19:984-93.

19 Brodersen R, Jacobsen J. Serum bilirubin diglucuronide in the human adult and newborn child. In: Bouchier ID, Billing BH, eds. Bilirubin metabolism. Oxford: Blackwell, 1967:111-5.

${ }^{20}$ Brodersen R. Aqueous solubility, albumin binding, and tissue distribution of bilirubin. In: Ostrow JD, ed. Bile pigments and jaundice. Molecular, metabolic, and medical aspects. Basel: Marcel Dekker, 1986:157-81.

21 Ritter DA, Kenny JD, Norton HJ, Rudolph AJ. A prospective study of free bilirubin and other risk factors in the development of kernicterus in premature infants. Pediatrics 1982;69:260-6.

22 Brodersen R, Friis-Hansen B, Stern L. Drug induced displacement of bilirubin from albumin in the newborn. Dev Pharmacol Ther 1983;6:217-29.

${ }^{23}$ Stern L, Denton RL. Kernicterus in small premature infants. Pediatrics 1965;35:483-5.

${ }^{24}$ Brodersen R, Stern L. Kernicterus research and the basic sciences. A prospect for future development. Pediatrics 1987;79:154-6.

${ }_{25}$ Maynard EA, Schultz RL, Pease DC. Electron microscopy of the vascular bed of rat cerebral cortex. Am J Anat 1957;100: 409-34.

Correspondence and requests for reprints to Dr A Knudsen, Kattegatsvej 38, DK-9700 Brønderslev, Denmark.

Accepted 3 October 1988 